\title{
Notas sobre a presença dos gatos domésticos nas cidades
}

médias

\section{Notas sobre la presencia de los gatos domésticos en las ciudades medias}

\section{Notes on the presence of domestic cats in medium-sized cities}

\author{
Juciane Maria Johann \\ jucijohann@yahoo.com.br \\ Universidade Federal de Mato Grosso, UFMT, campus de Rondonópolis, MT \\ Fabio Angeoletto \\ fabio_angeoletto@yahoo.es \\ Universidade Federal de Mato Grosso, UFMT, campus de Rondonópolis, MT \\ Enrique Richard \\ chelonos@gmail.com \\ Escola Superior Politécnica Agropecuária de Manabí, Equador
}

\begin{abstract}
Resumo: A ampliação das áreas urbanas está levando as pessoas a adotarem gatos como animais de companhia porque são mais ajustados ao estilo de vida moderno, demandam menores espaços e podem ficar sozinhos por períodos maiores em comparação com outros animais, como o cão. Porém, ambientalistas estão preocupados com o aumento da população de felinos nos meios urbanos, já que acreditam que os gatos ameaçam a vida de aves e pequenos mamíferos selvagens e que podem chegar a extinguir esses animais. Esta nota de pesquisa apresenta opiniões pró e contra à presença de gatos em cidades e como manejá-los. O manejo correto desses animais é necessário, pois o maior risco ambiental relacionado à presença dos felinos está na reprodução descontrolada desses animais e consequente prática de abandono de filhotes indesejáveis por parte de seus tutores.
\end{abstract}

Palavras-chave: Urbanização; impactos ambientais; predação; controle populacional; ecologia urbana.

Resumen: La ampliación de las áreas urbanas está llevando personas a adoptar gatos como mascotas, ya que se ajustan más al estilo de vida moderno, demandan espacios más pequeños y pueden quedarse solos por períodos más largos en comparación con otros animales, como perros. Sin embargo, los ambientalistas están preocupados por el aumento de la población de felinos en zonas urbanas, puesto que creen que los gatos amenazan la vida de aves y pequeños mamíferos silvestres y pueden llegar a extinguir a estos animales. Esta nota e investigación presenta opiniones sobre los pros y los contras la presencia de gatos en las ciudades y cómo manejarlos. El manejo correcto de estos animales es necesario, pues el mayor riesgo ambiental relacionado 
con la presencia de los felinos es la incontrolada reproducción de estos animales y la consecuente práctica de abandono de gatitos indeseables por sus tutores.

Palabras clave: Urbanización; impactos ambientales; predación; control poblacional; ecología urbana.

\begin{abstract}
The expansion of urban areas is leading people to adopt cats as pets as they are better adapted to the modern lifestyle, require smaller spaces and could stand alone for longer periods compared to other animals such as dogs. However, environmental groups are concerned about the increasing population of felines in urban areas, as they believe that cats threaten the lives of wild birds and small mammals that may be extinct. This research note presents pros and cons of cats' presence in cities as how to handle them. Correct management of these animals is necessary, since the greatest environmental risk related to their presence is their uncontrolled reproduction, and consequent abandoning of undesirable pups by their tutors.
\end{abstract}

Keywords: Urbanization; environmental impacts; predation; population control; urban ecology.

\title{
O QUE SÃO CIDADES MÉDIAS: UMA BREVÍSSIMA CONTEXTUALIZAÇÃO
}

Apesar da expressão 'cidades médias' ser amplamente utilizada, sua definição ainda é matéria de debates. A população e outras características utilizadas para a classificação dessas cidades variam muito conforme os autores. Conte (2013), para citar um exemplo, conceitua as cidades médias como as urbes consideradas de grande valor estratégico para o desenvolvimento regional, considerando sua posição geográfica na rede urbana, dados socioeconômicos e demográficos e função dentro da macrorregião em que se localiza.

O critério demográfico tem sido um dos mais utilizados para a classificação do tamanho das cidades, no entanto, os limites populacionais que determinam as cidades como sendo pequenas ou médias diferem muito nos diversos países do mundo, bem como dentro do espaço territorial do Brasil. Possuindo o país uma heterogeneidade muito grande em seu espaço, então varia a determinação do tamanho da população como fator classificatório das cidades (AMORIM FILHO, 2002; RIGOTTI, 2016).

A Organização das Nações Unidas (ONU) considera que cidades médias são aquelas que possuem entre 100 mil e 3 milhões de habitantes, já o VII Congresso Ibero Americano de Urbanismo considerou como sendo cidades médias todas aquelas que contenham de 20 mil a 500 mil habitantes, enquanto a Comissão Europeia define como cidades médias aquelas que possuem entre 100 e 250 mil habitantes (CONTE, 2013).

Embora o critério demográfico para a definição de cidades médias não seja universalmente aceito - vide, por exemplo, as críticas de Costa (2002) e Pereira (2004), Serra (1998) propôs o intervalo entre 100 mil e 500 mil habitantes para classificar as cidades médias do Brasil. Esse intervalo reúne centros urbanos que têm escala suficiente para a geração de economias de aglomeração, como atividades industriais, mas que ainda não são significativamente afetados por deseconomias, como os congestionamentos, típicos das grandes cidades. Este critério é adotado pelo IBGE para diferenciar as cidades brasileiras de médio porte das pequenas (ANGEOLETTO, 2012). 
A partir dos anos 1990 o Brasil passou a ter um aumento considerável no número de cidades médias; atualmente existem 264 cidades médias no Brasil e elas concentram 25\% de toda a população do país (ANGEOLETTO et al., 2016). As cidades médias são locais oportunos para os investimentos de capitais de diversas atividades e passam a ofertar bens e serviços aos habitantes da área em que influencia, transformando-se em espaços de consumo locais e regionais. Elas passaram a ser valorizadas como fator de equilíbrio para redes urbanas onde a distância entre cidades grandes e pequenas são mais acentuadas, como ocorre no Brasil (CONTE, 2013).

\section{CONHECENDO RONDONÓPOLIS, MATO GROSSO}

Rondonópolis é uma cidade de porte médio com população estimada de 228.857 habitantes e densidade demográfica de $47 \mathrm{hab} / \mathrm{km}^{2}$ (IBGE, 2018), situada na mesorregião

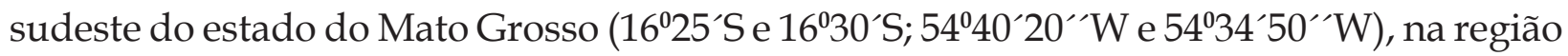
Centro-Oeste do Brasil (CUTRIM; CAMPOS, 2010).

Grande parte do desenvolvimento econômico de Rondonópolis deve-se a instalação de grandes indústrias na cidade, mas empresas de transportes e exportadoras de grãos contribuem e conferem ao município o título de segunda maior exportadora de grãos do estado. Além disso, a população conta com um variado comércio e implantação de redes atacadistas e varejistas, além de possuir uma quantidade significativa de universidades públicas e privadas e estabelecimentos no setor de saúde que conferem ao município o título de polo regional de educação e saúde (SANTOS et al., 2014). Esses aspectos garantem a Rondonópolis a segunda economia do estado e uma das 100 maiores do país com um PIB per capita de R\$43.024,92 (IBGE, 2018).

Segundo o IBGE (2018), Rondonópolis apresentou uma taxa de crescimento populacional de 2,94\% entre os anos de 2017 e 2018, sendo a maior em crescimento populacional do estado do Mato Grosso.

Rondonópolis está inserida no bioma Cerrado, um hotspot global de biodiversidade (SILVA et al., 2017). Ainda que estudos sobre a ecologia e a biodiversidade de cidades brasileiras sejam escassos (ANGEOLETTO; SANTOS, 2015), há alguns indícios de que a zona urbana rondonopolitana abrigue espécies da fauna do Cerrado. Um levantamento recente da avifauna, um fragmento florestal completamente encapsulado por uma matriz urbana, quantificou 127 espécies de aves: 15\% da avifauna do Cerrado (TOLEDO et al., 2018). A imensa e veloz degradação desse bioma megadiverso (daí sua condição de hotspot de biodiversidade) torna qualquer área possível, inclusive as cidades, de compor espaços preciosos para a conservação da biodiversidade.

\section{A POPULAÇÃO DE FELINOS AUMENTA NAS CIDADES, E ISSO DEMANDA GESTÃO AMBIENTAL}

A urbanização historicamente traduziu-se em impactos ambientais negativos e nas cidades médias do Brasil a expansão urbana acontece sem um suporte adequado 
de planificação e gestão urbanas (ANGEOLETTO et al., 2016). Recentemente ecólogos urbanos vêm estudando os impactos das populações urbanas de gatos domésticos (Felis catus). Esses cientistas argumentam que os felinos são uma grave ameaça à biodiversidade, principalmente de espécies de animais como aves, répteis e pequenos mamíferos silvestres (THOMAS; BAKER; FELLOWES, 2014).

Com a verticalização das cidades, envelhecimento da população humana e a mudanças no estilo das pessoas, há uma crescente demanda por animais de estimação que estejam adaptados a ambientes menores e que possuam menos necessidades de cuidados por parte dos tutores. Esses fatores têm sido determinantes para um aumento contínuo no número de felinos nas residências brasileiras (MARQUES; OLIVEIRA; GOMES, 2017).

A população de gatos em domicílios brasileiros foi estimada em 22,1 milhões, e a de cães, em 52,2 milhões. Atualmente, em alguns países, a população de gatos domésticos já ultrapassa a de cães (GUERRA, 2016) e, de acordo com o IBGE (2013), o Brasil segue esta tendência: sua população canina cresce aproximadamente $4 \%$, e a felina $8 \%$, ao ano. Em um futuro breve a população felina igualar-se-á a canina no Brasil (IBGE, 2013).

Nos últimos anos, vários estudos analisaram o potencial predatório e consequente ameaça que gatos domésticos representam à vida de animais silvestres, principalmente aves, artrópodes, pequenos mamíferos e répteis. Os gatos são carnívoros obrigatórios e caçam mesmo quando bem alimentados, e ao longo da evolução a partir de seu ancestral mantiveram sua elevada eficiência de caça (KERBER, 2017). Essas características apontam esses animais como potencial ameaça e uma das principais causas do declínio de outras espécies em diferentes áreas do mundo (FERREIRA, 2016). Estima-se que os gatos domésticos foram os responsáveis diretos pela extinção de ao menos 63 espécies de vertebrados (LOSS; MARRA, 2017).

Em regiões urbanas com intensa concentração de gatos é possível que estes animais exerçam impacto sobre populações selvagens tanto pela atividade predatória quanto pela transmissão de doenças, pois são vetores para vários agentes etiológicos, disseminando estes patógenos tanto para o ser humano quanto para outras espécies (KERBER, 2017). Outra perspectiva com relação aos impactos causados pela presença deste felinos, especialmente os abandonados, é a possibilidade de uma competição com espécies nativas que se alimentam das mesmas presas, comprometendo assim a sobrevivência destas espécies (FERREIRA, 2016).

Em um estudo de monitoramento de gatos domésticos realizado na cidade de Reading, na Inglaterra, Thomas e colaboradores (2014) concluíram que os gatos passavam a maior parte do tempo em jardins e áreas verdes da cidade. Uma das hipóteses levantadas foi a de que os animais se deslocassem para esses locais com o objetivo de procurar presas, e este estudo sugeriu que seria eficaz para um equilíbrio entre a conservação da biodiversidade e a presença de gatos domésticos, se os mesmos fossem mantidos permanentemente a uma distância de 300 a 400 metros de distância das áreas de conservação ambiental como parques ecológicos, por exemplo, o que já vem sendo realizado por algumas empreiteiras, através da proibição da posse de gatos por moradores em empreendimentos imobiliários próximos a estas áreas. 
Em relação à saúde dos seres humanos, os felinos podem representar uma fonte de infecção de agentes zoonóticos (aqueles que são capazes de produzir doenças em seres humanos), principalmente quando esses animais não recebem cuidados de saúde preventivos, como ocorre com os animais abandonados. Os gatos podem ser responsáveis pela transmissão de agentes que geram problemas de pele aos humanos (dermatofitoses); doenças gastrointestinais como salmonelose, giardiose, dipilidiose e tricuríase; infecções fúngicas e protozoárias como criptosporidiose, cistoisosporidiose, toxocaríase e toxoplasmose (esta com consequências graves para o feto, podendo causar cegueira e aborto). Os gatos domésticos também transmitem doenças virais como a raiva, a qual provoca distúrbios neurológicos e é altamente mortal em humanos (ALVES, 2016).

Os impactos à saúde humana, e principalmente os impactos à biodiversidade provocados pelos gatos domésticos, têm gerado debates entre gestores, ambientalistas e cientistas. Militantes ambientalistas e cientistas têm sugerido eliminar os gatos, principalmente em ambientes insulares (VEITCH, 2001; WOOD; TERSHY; HERMOSILLO, 2002). Na outra ponta do ambientalismo, há também uma intensa guerra de informação e contrainformação promovidas por ativistas dos direitos animais. A Alley Cats Allies, por exemplo, uma organização com mais de 650.000 membros, atua sistematicamente para desacreditar cientistas e resultados de investigações cientificas que demonstrem impactos causados pelos felinos, e para impedir quaisquer politicas de conservação biológica que restrinjam a livre movimentação dos gatos (LOSS; MARRA, 2018). A ironia, nesses debates onde a desonestidade intelectual medra, é que os defensores dos direitos dos gatos não entendem, ou não querem entender, que a mesma lógica deveria ser estendida aos animais silvestres que frequentam os ecossistemas urbanos: pássaros silvestres não merecem também viver e transitar por nossos quintais, praças e parques?

\section{ROTULAR GATOS COMO VILÕES OU VÍTIMAS NÃO É O MELHOR CAMINHO PARA MANEJA-LOS}

Os impactos dos gatos não são graves apenas em relação à diversidade biológica urbana. Gatos predam milhões de vertebrados anualmente, em zonas rurais (KRAUZEGRYZ; GRYZ; ŻMIHORSKI 2019). eliminar os gatos das paisagens antropogênicas poderia ser uma solução para seus danos sobre a biodiversidade. Mas, excetuando-se ilhas e ambientes insulares, isso seria desejável? Não, se incluirmos os seres humanos nesse debate. Há vários benefícios que o convívio com gatos traz à saúde humana. O contato entre gatos e humanos melhora o convívio social entre as pessoas e reduz o estresse e a ansiedade (BEETZ et al., 2012). Há benefícios cardiovasculares, pois desfrutar da companhia felina combate a hipertensão (ALLEN, 2003). Gatos potencializam as respostas ao tratamento e diminuem o tempo de internação, quando em contato com humanos hospitalizados (HALM, 2008). Eles aumentam a eficácia de terapias que motivam crianças autistas a se socializarem com demais humanos (SAMS, FORTNEY; WILLEMBRING, 2006). Ademais crianças que convivem com cães e gatos no início da vida desenvolvem melhor o seu sistema 
imunológico e diminuem os riscos de infecções respiratórias durante a vida (BERGROTH et al., 2012). Outro argumento importante é de que, a maioria dos seres humanos vivendo em cidades, o contato com animais 'urbanos', como gatos, é uma das poucas oportunidades de uma relação biofílica com a biodiversidade para crianças cada vez mais isoladas em paisagens de concreto (DUNN et al., 2006).

Nós estamos monitorando a movimentação de gatos domésticos na zona urbana de Rondonópolis com GPS trackers, baseados no método descrito em Thomas, Baker e Fellowes (2014) e Hanmer, Thomas e Fellowes (2017). Os gatos monitorados dividem-se em castrados e não castrados, machos e fêmeas. Nossa hipótese é a de que gatos deslocamse principalmente por espaços urbanos onde ocorrem animais silvestres, como quintais e parques, e os primeiros resultados (monitoramos 20 gatos entre os meses de novembro de 2018 a abril de 2019) apontam nessa direção.

Então, como manejá-los? Alguns estudos científicos apontam um resultado favorável do método CCD - captura, castração e devolução ao ambiente, tanto pela redução da população quanto pelas modificações de comportamento que os gatos apresentam após serem castrados. Ferreira (2016) demonstrou que a prática de castração reduz a área e o padrão de atividade dos gatos e, consequentemente, diminui o potencial de disseminação de doenças e, indiretamente, ocorrerá a redução da predação por parte desses animais, e, ainda, a taxa de sobreposição dos felinos domésticos e silvestres. Concordando com essa ideia, Nogueira (2018) concluiu que a esterilização de machos contribui significativamente para a redução ou inibição de alguns comportamentos como perambulação e agressividade. A castração também é benéfica pois evita o crescimento desordenado da população de gatos, o que estimula o abandono e consequente formação de populações ferais. Faria (2014) conseguiu comprovar a diminuição do abandono, e, consequentemente do número de animais perambulando pelas ruas de um bairro de. A autora relata que o método CCD vem sendo utilizado em muitos países, inclusive no Brasil, nos últimos anos e tem demonstrado resultados positivos na manutenção da população de gatos estável.

Há uma vantagem adicional do manejo CCD. Uma vez que os gatos castrados são devolvidos ao seus ambientes de origem, Como são animais territoriais, isso evita que machos não castrados colonizem esses territórios, e procriem. De acordo com Levy, Isaza e Scott (2014), o método de manejo CCD contribui para a redução das colônias de gatos. A eficiência desse método, por outro lado, tem sido questionada por parte da comunidade científica (vide por exemplo LOSS; MARRA, 2018)

\section{CONSIDERAÇÕES FINAIS}

Debates a parte, é um fato que os gatos domésticos são animais que apresentam um risco ambiental bastante grande, principalmente devido ao seu comportamento relacionado a caça. Mas, apesar disso, sua presença apresenta inúmeros benefícios, não apenas relacionados à saúde humana, mas também, pelo próprio instinto de caçadores, acabam por controlar algumas pragas, como ratos e baratas, por exemplo. No entanto, 
acreditamos que o maior problema está relacionado ao crescimento desordenado da população de felinos, e talvez esta seja a verdadeira ameaça ao ambiente.

Se a presença de gatos nas cidades possui méritos, é necessário o monitoramento de seus movimentos pela cidade para compreender por onde os gatos circulam nas cidades, quanto tempo dispendem em áreas verdes urbanas. O conhecimento da ecologia espacial de gatos em ambientes urbanos é importante para a criação de legislação e políticas públicas municipais que ajudem a minimizar seus impactos. É recomendável que estudos como o realizado por Thomas e sua equipe (2014) e o que estamos realizando em Rondonópolis, sejam replicados nas cidades de países megadiversos, como o Brasil. As cidades precisam ser planejadas para que aumentem o suporte à biodiversidade. Não apenas os ambientes prístinos devem ser reservados à conservação biológica, mas as cidades também podem e devem incrementar sua capacidade de atrair e manter espécies silvestres (ANGEOLETTO; SANTOS, 2015; SHAFFER, 2018). Manter os gatos sob controle é uma das várias demandas de cientistas e gestores, em prol da conservação da biodiversidade urbana.

\section{REFERÊNCIAS}

ALLEN, K. Are pets a healthy pleasure? The influences of pets on blood pleasure. Current directions in psychological science, v. 12, n. 6, p. 236-239, 2003.

ALVES, M.T.A. Parasitas Gastrointestinais em Gatos. Vila Real, 2016. Dissertação (Mestrado em Medicina Veterinária) - Universidade de Trás-os-Montes e Alto Doro, Portugal.

AMORIM FILHO, O.B.; RIGOTTI, J.I.R. Os limiares demográficos na caracterização das cidades médias. In: ENCONTRO NACIONAL DE ESTUDOS POPULACIONAIS, 13., 2002. Caxambu. Anais...Caxambu: ABEP, 2002. v.1, p. 1-22

ANGEOLETTO, F. Planeta cidade: ecologia urbana e planejamento de cidades médias do Brasil. Madri, 2012. Tese (Doutorado em Ecologia e Meio Ambiente) - Universidade Autônoma de Madrid.

ANGEOLETTO, F.; SANTOS, J.W.M.C. Los biólogos brasileños no habitan en el planeta ciudad: por qué es urgente formar ecólogos urbanos. Revista Espaço Acadêmico, v. 14, n. 165, p. 74-82, 2015.

ANGEOLETTO, F. et al. Tipología sócio-ambiental de las ciudades medias de Brasil: aportes para un desarrollo urbano sostenible. URBE - Revista Brasileira de Gestão Urbana, v. 8, n. 2, p. 272-287, 2016.

BEETZ, A. et al. Psychosocial and psychophysiological effects of human-animal interactions: the possible role of oxytocin. Frontiers in Psychology: Psychology for clinical settings, v.3, article 234, 2012.

BERGROTH, E. et al. Respiratory tract illnesses during the first year of life: effect of dog and cat contacts. Pediatrics, v.130, n. 2, p. 1-10, 2012.

CONTE, C.H. Cidades médias: discutindo o tema. Sociedade e Território, v. 25, p. 45-61, 2013.

COSTA, E.M. Cidades Medias. Contributos para sua definição. Revista Finisterra, v. 23, p. 74, p. 101-128, 2002.

CUTRIM, A.O.; CAMPOS, J.E.G. Avaliação da vulnerabilidade e perigo à contaminação do Aqüífero Furnas na Cidade De Rondonópolis (MT) com aplicação dos métodos GOD e POSH. Geociências, v. 29, n. 3, p. 401-411, 2010.

DUNN, R.R. et al. The pigeon paradox: dependence of global conservation on urban nature. Conservation biology, v. 20, n. 6, p. 1814-1816, 2006. 
FARIA, J.A. Relação/controle populacional de cães e gatos/melhoria nas condições ambientais e bemestar da comunidade no bairro de Paupina em Fortaleza - Ceará. Mossoró, 2014. Dissertação (Mestrado em Ambiente, Tecnologia e Sociedade) - Universidade Federal do Semi-Árido, UFERSA.

FERREIRA, G.A. Gatos domésticos em ambiente insular da Mata Atlântica: potenciais impactos sobre mamíferos silvestres e a castração como estratégia para a conservação. Juiz de Fora, 2016. Tese (Doutorado em Ecologia Aplicada ao Manejo e Conservação de Recursos Naturais) - Universidade Federal de Juiz de Fora, UFJF.

GUERRA, I.C.L. Ecologia urbana do gato doméstico Felis silvestris catus na cidade de Barcelona. Lisboa, 2016. Dissertação (Mestrado em Medicina Veterinária) - Universidade Lusófona de Humanidades e Tecnologias, Portugal.

HALM, M.A. The healing power of the human-animal connection. American journal of critical care, v.17, n.4, p. 373-377, 2008.

HANMER, H.J.; THOMAS, R.L.; FELLOWES, M.D. Urbanization influences range size of the domestic cat (Felis catus): consequences for conservation. Journal of Urban Ecology, v. 3, n. 1, p.jux014, 2017.

IBGE - Instituto Brasileiro de Geografia e Estatística. Pesquisa Nacional de Saúde. 2013. Disponível em: http:/ / www.ibge.gov.br/PNS/2013/pns.2013.pdf. Acesso em: 12 jan. 2019.

IBGE - Instituto Brasileiro de Geografia e Estatística. Panorama das cidades. 2018. Disponível em: http:/ / www.cidades.ibge.gov.br/brasil/mt/rondonopolis/panorama. Acesso em: 22 fev. 2019.

KERBER, S.S. Predação por gatos domésticos (Felis catus) em ambiente urbano, Florianópolis, Santa Catarina. Florianópolis, 2017. Dissertação (Mestrado em Ciências Biológicas) - Universidade Federal de Santa Catarina, UFSC.

KRAUZE-GRYZ, D.; GRYZ, J.; ŻMIHORSKI, M. Cats kill millions of vertebrates in Polish farmland annually. Global Ecology and Conservation, v. 17, p. 51-59, 2019.

LEVY, J.K.; ISAZA, N.M.; SCOTT, K.C. Effect of high-impact targeted trap-neuter-return and adoption of community cats on cat intake to a shelter. The Veterinary Journal, n. 201, p. 269 -274, 2014.

LOSS, S.R.; MARRA, P.P. Population impacts of free-ranging domestic cats on mainland vertebrates. Front. Ecol. Environ., v.25, n. 9, p.502-509, 2017.

LOSS, S.R.; MARRA, P.P. Merchants of doubt in the free-ranging cat conflict. Conservation Biology, 32, 265-266, 2018.

MARQUES, S.M.T; OLIVEIRA, M.R.F.; GOMES, M.J.T.M. Parasitos gastrintestinais em gatos da cidade de Porto Alegre, Rio Grande do Sul. Pub. Vet, v.11, n.11, p.1132-1137, 2017.

NOGUEIRA, V.M. Efeitos da orquiectomia sobre o comportamento e surgimento de afecções urinárias em felinos domésticos. Areia, 2018. Monografia (Bacharelado em Medicina Veterinária) - Universidade Federal da Paraíba, UFP.

PEREIRA, J.C.M. Importância e significado das cidades médias na Amazônia: uma abordagem a partir de Santarém (PA). Belém, 2004. Dissertação (Mestrado em Desenvolvimento Sustentável do Trópico Úmido) - Universidade Federal do Pará, UFPA.

SAMS, M.J.; FORTNEY, V.E.; WILLEMBRING, S. Occupational therapy incorporating animals for children with autism: a pilot investigation. American Occupational Therapy Association, v. 60, p. 268-274, 2006.

SHAFFER, H. Bradley. Urban biodiversity arks. Nature Sustainability, v. 1, n. 12, p. 725, 2018.

SANTOS, A.P.A. et al. Perfil Rondonópolis: geografia, demografia e economia. 6.ed. Rondonópolis: ACIR/ Prefeitura municipal, 2014.

SERRA, R.V. Cidades médias brasileiras: um recente retrato econômico e populacional. Rio de Janeiro, 1998. Dissertação (Mestrado em Planejamento Urbano e Regional) - Universidade Federal do Rio de Janeiro, UFRJ.

SILVA, N. et al. The negative influences of the new Brazilian Forest Code on the conservation of riparian forest. European Journal of Ecology, v.3, n.2, p. 116-122, 2017. 
THOMAS, R.L.; BAKER, P.J.; FELLOWES, M.D.E. Ranging characteristics of the domestic cat (Felis catus) in an urban environment. Urban Ecosyst., n. 17, p. 911-921, 2014.

TOLEDO, A. et al. Estudo técnico para a criação de Unidade de Conservação em Rondonópolis, MT. Rondonópolis: PM/ UFMT, 2018.

VEICHT, C.R. The eradication of feral cats (Felis catus) from Little Barrier Island, New Zeland. New Zeland Journal of Zoology. v. 28, p. 1-12, 2001.

WOOD, B., TERSHY, B.R., HERMOSILLO, M.A., et al. Removing cats from islands in northwest Mexico. In: VEITCH, C.R.; CLOUT, M.N. (Eds). Turning the Tide: The Eradication of Invasive Species. Gland: IUCN SSC Invasive Species Specialist Group, 2002.p. 374-380.

Data de submissão: 13/mar./2019

Data de aceite: 22 / set./2019 